\title{
Patrones de prescripción de antidepresivos en pacientes afiliados al Sistema General de Seguridad Social en Salud de Colombia
}

\author{
Jorge E. Machado-Alba, ${ }^{1}$ Cristhian David Morales Plaza ${ }^{1}$ \\ y Mónica Johanna Solarte Gómez ${ }^{1}$
}

Forma de citar

Machado-Alba JE, Morales Plaza CD, Solarte Gómez MJ. Patrones de prescripción de antidepresivos en pacientes afiliados al Sistema General de Seguridad Social en Salud de Colombia. Rev Panam Salud Publica. 2011;30(5):461-8.

RESUMEN Objetivo. Determinar los patrones de prescripción de medicamentos antidepresivos en un grupo de afiliados al Sistema General de Seguridad Social en Salud de Colombia.

Métodos. Estudio descriptivo observacional con 9881 pacientes medicados con antidepresivos, de ambos sexos, mayores de 5 años, con tratamiento continuo de agosto a octubre de 2009 y residentes en 56 ciudades colombianas. Se diseñó una base de datos sobre consumo de medicamentos, obtenidos por la empresa que distribuye los medicamentos a los pacientes.

Resultados. Edad promedio de 59,1 $\pm 16,1$ años; $73,7 \%$ de los participantes fueron mujeres. Del total de pacientes, $83,3 \%$ recibían monoterapia y 16,7\% dos o más antidepresivos. El orden de prescripción de los medicamentos fue: inhibidores selectivos de la recaptación de serotonina $47,0 \%$, atípicos $37,8 \%$, tricíclicos $31,8 \%$, inhibidores selectivos de la recaptación de serotonina y norepinefrina 1,8\% e inhibidores selectivos de la recaptación de norepinefrina $0,03 \%$. Las combinaciones más empleadas fueron fluoxetina + trazodona $(\mathrm{n}=1029)$, amitriptilina + fluoxetina $(\mathrm{n}=265)$, amitriptilina + trazodona $(\mathrm{n}=122)$, fluoxetina + imipramina $(\mathrm{n}=106)$ e imipramina + trazodona $(\mathrm{n}=71)$. Las comedicaciones más prescritas fueron antihipertensivos $(52,3 \%)$, hormona tiroidea $(23,3 \%)$, antiinflamatorios $(19,6 \%)$, antiepilépticos $(15,4 \%)$, antidiabéticos $(13,8 \%)$, ansiolíticos e hipnóticos $(12,4 \%)$, antipsicóticos $(7,4 \%)$, antiparkinsonianos $(4,3 \%)$ y antineoplásicos $(2,2 \%)$.

Conclusiones. Predominan hábitos de prescripción de medicamentos de alto valor terapéutico, principalmente en monoterapia antidepresiva. La mayoría de los antidepresivos se emplean en dosis menores que las recomendadas. Se plantea la necesidad de diseñar estrategias educativas para corregir algunos hábitos de prescripción e investigaciones que evalúen la efectividad del tratamiento.

Palabras clave Trastorno depresivo; agentes antidepresivos; prescripciones de medicamentos; amitriptilina; fluoxetina; imipramina; trazodona; Colombia.

La depresión es un trastorno de prevalencia creciente que afecta al individuo

Grupo de Investigación en Farmacoepidemiología y Farmacovigilancia. Departamento de Ciencias Básicas, Facultad de Ciencias de la Salud, Universidad Tecnológica de Pereira, Pereira, Colombia. La correspondencia se debe enviar a Jorge E. Machado-Alba, machado@utp.edu.co en su funcionamiento biológico, psicológico y social, y constituye un grave problema de salud pública. Según la Organización Mundial de la Salud (OMS) dicho trastorno representó en el año 2002 una carga de morbilidad de 4,5\%. Adicionalmente, incrementa el riesgo de suicidio entre quienes la padecen $(1,2)$. La prevalencia en las Américas varía entre 4,5\% y 13,2 \% y la frecuencia del trastorno se incrementa en pacientes hospitalizados (3-6). La prevalencia en Colombia ha variado entre $12,1 \%$ y $19,6 \%(2,7)$ y es más común en mujeres $(6,8)$.

Las formas más comunes de la enfermedad son el trastorno depresivo mayor, la distimia y la fase depresiva del tras- 
torno afectivo bipolar $(9,10)$. Los medicamentos antidepresivos se clasifican en antidepresivos tricíclicos, inhibidores selectivos de la recaptación de serotonina (ISRS), inhibidores selectivos de la recaptación de norepinefrina, inhibidores selectivos de la recaptación de serotonina y norepinefrina, antidepresivos atípicos e inhibidores de la monoaminooxidasa (IMAO) (11). La selección inicial se basa en gran medida en la seguridad y tolerabilidad del medicamento, las respuestas anteriores, las comorbilidades médicas o psiquiátricas, las posibles interacciones y el costo. La American Psychiatric Association recomienda usar inicialmente ISRS en la mayoría de los pacientes, y esa recomendación es acogida por muchos prescriptores en nuestro país por falta de guías nacionales (12).

Las comorbilidades generales que se asocian más frecuentemente con la depresión son enfermedad coronaria $(14,0$ $47,0 \%)(13)$, insuficiencia cardíaca congestiva $(60,0 \%)(14)$, ictus $(38,0 \%)(15)$, hipertensión $(53,0 \%)(16)$, cáncer $(38,0 \%)$ (17), enfermedad de Parkinson, $(37,0 \%)$ (18), VIH/sida $(37,0 \%)$ (19), hipotiroidismo $(16,0 \%)(20)$, diabetes $(9,0 \%)(21-$ $23)$, artritis reumatoide $(25,0 \%$ a $40,0 \%)$ (24) y epilepsia $(26,7 \%)$ (25). Las comorbilidades psiquiátricas más frecuentes son la esquizofrenia $(13,3 \%$ de los pacientes con depresión) (26), la crisis de angustia $(31,4 \%)$, el trastorno obsesivo-compulsivo (entre $8,7 \%$ y $21,1 \%$ ) y el trastorno de ansiedad generalizada $(37,1 \%)(27)$. Además, se ha encontrado una asociación entre los trastornos del sueño y el riesgo de desarrollar un episodio depresivo mayor (28).

Uno de los grandes retos que debe enfrentar el Sistema General de Seguridad Social en Salud (SGSSS) es el mejoramiento en la detección y el acceso al tratamiento, y el incremento de las tasas de control de los pacientes depresivos. En 1995 se creó en Colombia el Instituto Nacional para la Vigilancia de Medicamentos y Alimentos (INVIMA) y dentro de un marco de uso adecuado de productos farmacéuticos se adoptó un listado de medicamentos esenciales del Plan Obligatorio de Salud (POS), que incluye cinco medicamentos antidepresivos: amitriptilina, imipramina, fluoxetina, trazodona y tranilcipromina (29). Para acceder a otros medicamentos antidepresivos, los prescriptores deben hacer solicitudes especiales a través de mecanismos establecidos por las institu- ciones prestadoras de servicios de salud, denominados Comités Técnico-Científicos (CTC), donde el médico tratante fundamenta la necesidad de usar un medicamento no incluido en el listado POS (29); los pacientes también tienen un mecanismo legal (tutela) para solicitar el acceso a un medicamento excluido del listado. La implantación de sistemas de información sobre prescripciones realizadas por los médicos ha sido fundamental para contribuir al uso racional de medicamentos (30).

Con el objeto de describir los patrones de prescripción de los medicamentos antidepresivos y de la comedicación empleada con mayor frecuencia en estos pacientes, se llevó a cabo esta investigación en personas afiliadas al SGSSS en Colombia que consumían dichos medicamentos.

\section{MATERIALES Y MÉTODOS}

Se realizó un estudio descriptivo observacional sobre los hábitos de prescripción de medicamentos antidepresivos en una población aproximada de 3,7 millones de personas afiliadas al régimen contributivo del SGSSS en tres Empresas Promotoras de Salud (EPS), que corresponde aproximadamente al $20,3 \%$ de la población afiliada activa de este régimen en el país y al 8,2\% de la población colombiana. Se analizaron los datos de prescripción a partir de prescripciones dispensadas desde el 1 de agosto hasta el 31 de octubre de 2009 a 9881 pacientes tratados con antidepresivos, inscritos durante los últimos tres meses en alguna de las 56 ciudades colombianas en las que se dispone de bases de datos confiables, que tienen entre 30 mil y 7,5 millones de habitantes. ${ }^{3}$

Se incluyeron los datos de individuos con dispensación de antidepresivos de todas las edades, de ambos sexos, atendidos en consulta médica y cuyo tratamiento antidepresivo se mantuvo durante los tres meses. Este requisito tiene

\footnotetext{
3 Ciudades donde se realizó la recolección de la información: Acacias, Armenia, Barranquilla, Bello, Bogotá, Bucaramanga, Buga, Cali, Cartagena, Cartago, Chía, Chinchiná, Ciénaga, Cúcuta, Dosquebradas, Envigado, Facatativá, Floridablanca, Funza, Fusagasugá, Girardot, Girardota, Girón, Ibagué, Itagüí, Jamundí, La Dorada, La Tebaida, La Virginia, Madrid, Manizales, Medellín, Montenegro, Montería, Neiva, Orito, Palmira, Pasto, Pereira, Popayán, Puerto Tejada, Quimbaya, Salamina, San José del Guaviare, Santa Marta, Santa Rosa de Cabal, Sevilla, Sincelejo, Soacha, Soledad, Tenjo, Tuluá, Valledupar, Villamaria, Villavicencio, Zipaquirá.
}

como objeto garantizar que los pacientes cumplieran el tratamiento de manera más o menos estable, lo que refleja la tolerancia a la medicación, de tal forma de excluir a las personas que incumplan las citas médicas al final del período de observación. Se considera que estos individuos introducen sesgos en un estudio dirigido a describir patrones de empleo de medicamentos utilizados de manera crónica y continua.

A partir de la información sobre los consumos de medicamentos obtenida de manera sistemática por la empresa que los dispensa (Audifarma S.A.) a la población afiliada, se diseñó una base de datos que permitió recoger los siguientes grupos de variables de pacientes con depresión: a) sociodemográficas (sexo, edad, ciudad); b) medicamentos antidepresivos dispensados en Colombia, con sus dosis respectivas (para la cuantificación de la dispensación se ha utilizado como unidad técnica de medida la dosis diaria definida, recomendada por la OMS como estándar internacional para la realización de estudios farmacoepidemiológicos). Entre los medicamentos se consideraron los antidepresivos tricíclicos, los ISRS, los inhibidores de la recaptación de norepinefrina, los inhibidores selectivos de la recaptación de serotonina y norepinefrina, los antidepresivos atípicos y los IMAO, que están disponibles en el mercado del país; c) la especialidad médica del prescriptor y d) si fueron prescritos en monoterapia o en terapia combinada.

Para la comedicación dispensada se tuvieron en cuenta todos los medicamentos pertenecientes a los siguientes grupos disponibles en el país: a) antihipertensivos; b) inotrópicos; c) antidiabéticos; d) antiinflamatorios no esteroideos, corticosteroides, antirreumáticos modificadores de la enfermedad; e) antiparkinsonianos; f) hormona tiroidea; g) antineoplásicos; h) antirretrovirales; i) antipsicóticos; j) anticonvulsivos y k) ansiolíticos e hipnóticos. La búsqueda de estos se basó en su correlación con las comorbilidades reportadas con mayor riesgo de depresión (13-28).

Se aceptó la comedicación como un indicador sustituto de la enfermedad crónica que ha sido asociada como factor de riesgo para que un paciente padezca depresión considerando las siguientes circunstancias: a) antihipertensivos/ hipertensión arterial; b) digitálicos/insuficiencia cardíaca; c) antiparkinsonia- 
nos/parkinson; d) antipsicoticos/esquizofrenia; e) anticonvulsivos/epilepsia; f) ansiolíticos e hipnóticos/ansiedad e insomnio; g) antiinflamatorios/artritis reumatoide, artrosis o enfermedad articular inflamatoria crónica; h) antirretrovirales/VIH-sida; i) antidiabéticos/ diabetes; j) antineoplásicos/cáncer y k) hormona tiroidea/hipotiroidismo. En tales casos se analizó la conveniencia o no del tipo de antidepresivo elegido con base en los efectos farmacológicos que puedan tener estos medicamentos en la comorbilidad. El protocolo se sometió a la aprobación del Comité de Ética Médica de la Facultad de Ciencias de la Salud de la Universidad Tecnológica de Pereira y a los Comités de Ética de las EPS, en la categoría de "investigación sin riesgo", según la resolución No. 008430 de 1993 del Ministerio de Salud de Colombia, que establece las normas científicas, técnicas y administrativas para la investigación en salud (31).

La base de datos fue revisada y validada por el Departamento de Farmacoepidemiología de la empresa encargada de la dispensación farmacéutica. Para el análisis de los datos, se utilizó el paquete estadístico IBM SPSS Statistics, versión 19 (IBM, EE.UU.) para Windows. Se emplearon las pruebas $t$ de Student o ANOVA para la comparación de variables cuantitativas y la de ji al cuadrado para las categóricas. Se aplicaron modelos de regresión logística binaria usando como variables dependientes la monoterapia/ politerapia antidepresiva y la comedicación (sí/no), y como covariables las que se asociaron significativamente con las variables dependientes en los análisis bivariados. Se determinó como nivel de significación estadística una $P<0,05$.

\section{RESULTADOS}

De los 9881 pacientes afiliados al SGSSS en tratamiento para la depresión por un período no menor de tres meses, correspondientes al $0,26 \%$ de la población afiliada a las EPS del estudio, la distribución por sexo mostró que 7290 (73,8\%) fueron mujeres y 2591 (26,2\%) varones, con una edad media de 59,1 \pm 16,1 años (rango 5-95 años); no hubo diferencias significativas en la edad entre varones y mujeres (varones 58,8 $\pm 17,4$; mujeres 59,3 $\pm 15,7 ; P=0,2$ ). En la figura 1 se muestra la distribución por edad y sexo.

En el cuadro 1 se resumen los patrones de prescripción de los medicamentos

FIGURA 1. Distribución de 9881 pacientes tratados con antidepresivos, por edada y sexo, Colombia, 2010

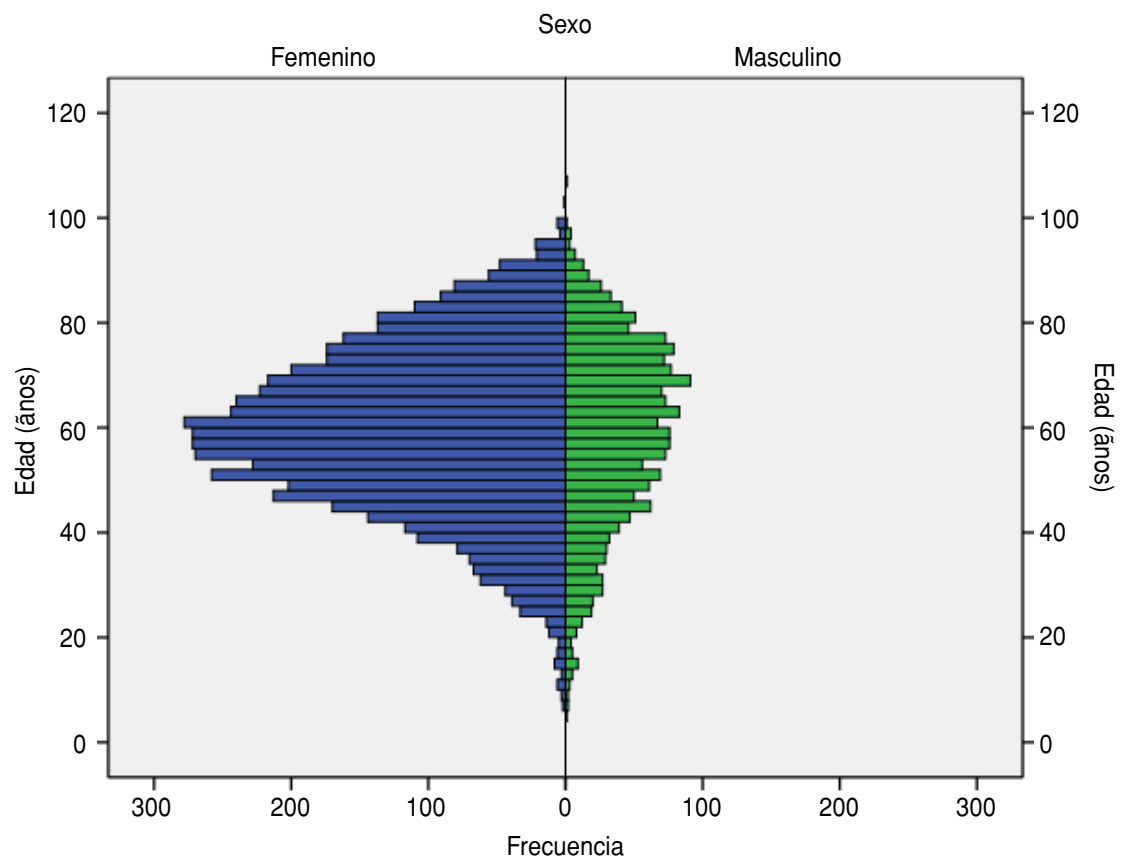

a $n$ por grupos de edad: menor de 10 años, $n=16$; de 10 a 25 años, $n=146$; de 26 a 59 años, $n=3$ 415; mayor de 60 años, $n=3558$.

CUADRO 1. Patrones de prescripción de antidepresivos del listado de medicamentos esenciales en Colombia, 2010

\begin{tabular}{|c|c|c|c|c|c|c|}
\hline \multirow[b]{2}{*}{ Medicamento } & \multicolumn{2}{|c|}{$\begin{array}{c}\text { Prescripciones/ } \\
\text { usuarios }\end{array}$} & \multicolumn{2}{|c|}{$\begin{array}{c}\text { Dosis } \\
\text { prescritas (mg/día) }\end{array}$} & \multirow{2}{*}{$\begin{array}{l}\text { Relación según } \\
\text { el sexo M:F }\end{array}$} & \multirow{2}{*}{$\begin{array}{c}\text { Edad } \\
(\text { media } \pm \mathrm{DE})\end{array}$} \\
\hline & No. & $\%$ & Media & Moda & & \\
\hline Fluoxetina & 3990 & 40,4 & 27,3 & 20 & $1: 3,2$ & $56,3 \pm 15,4$ \\
\hline Trazodona & 3625 & 36,7 & 61,7 & 50 & $1: 2,8$ & $62,6 \pm 15,5$ \\
\hline Amitriptilina & 2415 & 24,4 & 27,7 & 25 & $1: 3,1$ & $60,3 \pm 15,2$ \\
\hline Imipramina & 600 & 6,1 & 53,8 & 25 & $1: 2,8$ & $53,8 \pm 15,4$ \\
\hline Fluoxetina (jarabe) & 266 & 2,7 & 27,7 & 18,6 & $1: 2,5$ & $59,2 \pm 19,6$ \\
\hline Otros $^{b}$ & 694 & 7,8 & & & & \\
\hline
\end{tabular}

a:F, masculino:femenino. DE, desviación estándar.

b Otros: todos los demás antidepresivos dispensados (24 en total).

antidepresivos incluidos en el listado de medicamentos esenciales con los que se maneja con mayor frecuencia la depresión entre los pacientes afiliados al SGSSS en Colombia. Al considerarlos por grupos farmacológicos, de las 9881 prescripciones de medicamentos antidepresivos los más prescritos fueron ISRS $47,0 \%$, atípicos $37,8 \%$, tricíclicos $31,8 \%$, inhibidores selectivos de la recaptación de serotonina y norepinefrina 1,8\% e inhibidores selectivos de la recaptación de norepinefrina $0,03 \%$. Los medicamentos más empleados fueron fluoxetina, trazodona, amitriptilina e imipramina, de los cuales solo el primero estaba siendo usado a la dosis usual, mientras que los demás se prescribieron a dosis inferiores. Cabe resaltar que no se prescribió tranilcipromina y que las prescripciones de otros antidepresivos incluyeron bupropión, clomipramina, citalopram, doxepina, duloxetina, escitalopram, fluvoxamina, maprotilina, mirtazapina, paroxetina, reboxetina, sertralina y venlafaxina. Solo 2,8\% de las prescripciones fueron realizadas por un médico psiquiatra, y las restantes por un médico general de atención primaria.

\section{Monoterapia frente a asociaciones}

Del total de pacientes del estudio, a $8232(83,3 \%)$ se les prescribió un solo 
antidepresivo, en tanto que a 1649 $(16,7 \%)$ se les indicó una asociación de dos o más antidepresivos; esta diferencia fue estadísticamente significativa $(P<0,001)$. Hubo, incluso, 46 pacientes $(0,05 \%)$ que recibían tres medicamentos $\mathrm{y}$ uno tratado con cuatro antidepresivos. En la figura 2 se ilustra la relación monoterapia/politerapia para los antidepresivos que fueron evaluados; se destaca el hecho de que fue más probable emplearlos solos que en combinación. Entre los 1649 pacientes a quienes se les prescribieron asociaciones de antidepresivos, las utilizadas con mayor frecuencia fueron fluoxetina + trazodona $(n=1029 ; 62,4 \%$ de los individuos tratados con combinaciones), amitriptilina + fluoxetina $(n=265 ; 16,0 \%)$; amitriptilina + trazodona ( $n=122 ; 7,3 \%)$, fluoxetina + imipramina $(n=106 ; 6,4 \%)$ e imipramina + trazodona $(n=71 ; 4,3 \%)$.

Los pacientes mayores de 60 años y los tratados en las ciudades de Bogotá,
Medellín, Barranquilla, Bucaramanga y Cartagena tuvieron mayor probabilidad que los de otros grupos etarios y otras ciudades de ser manejados con monoterapia antidepresiva (cuadro 2 ).

\section{Comedicación}

La depresión frecuentemente está acompañada de comorbilidades, que necesitan de tratamiento adicional. Entre los pacientes incluidos, $8088(81,9 \%)$ recibían concomitantemente uno o varios de los siguientes grupos de medicamentos que reflejan comorbilidad y que podrían tener interacciones con algunos antidepresivos: antihipertensivos $(n=5166$; $52,3 \%$ de los pacientes); hormona tiroidea $(n=2298,23,3 \%)$, antiinflamatorios $(n=$ 1 939; 19,6\%); antiepilépticos $(n=1521$, $15,4 \%)$, antidiabéticos $(n=1365 ; 13,8 \%)$, ansiolíticos e hipnóticos $(n=1221$; $12,4 \%)$; antipsicóticos $(n=736 ; 7,4 \%)$; antiparkinsonianos $(n=425 ; 4,3 \%)$; antineo-

FIGURA 2. Frecuencia de prescripción de antidepresivos en monoterapia o politerapia, Colombia, 2010

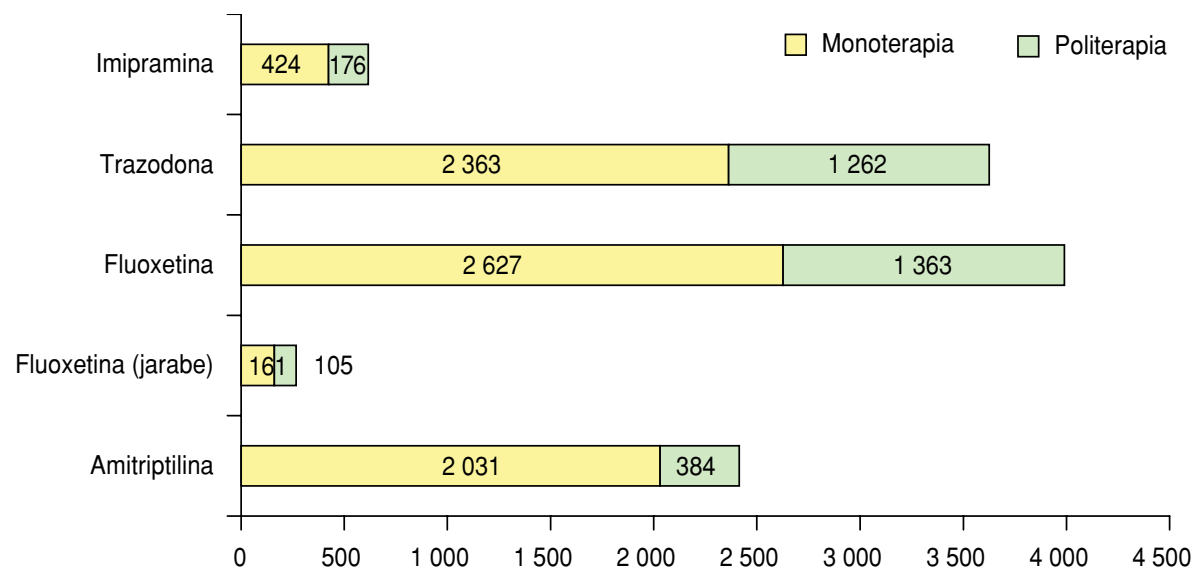

plásicos $(n=221 ; 2,2 \%)$ y antirretrovirales $(n=20 ; 0,2 \%)$. Se encontró un total de $177(1,7 \%)$ pacientes que recibían concomitantemente cuatro psicofármacos e incluso $31(0,3 \%)$ pacientes que recibían al mismo tiempo cinco psicofármacos, lo que incrementa el riesgo de interacciones sobre el sistema nervioso central y sobre el sistema nervioso autónomo.

$\mathrm{Al}$ analizar mediante regresión logística binaria la relación entre el empleo de comedicación y otras variables, se encontró que la única que se encontraba asociada significativamente con el empleo de comedicación fue la covariable "ciudad"; en particular, las ciudades de Bogotá, Cali y Barranquilla presentaron una asociación estadísticamente significativa con el uso de comedicación en sus pacientes (cuadro 3). No hubo diferencias por edad y sexo.

\section{Comparación entre ciudades}

Se compararon las variables demográficas y algunos indicadores de prescripción entre las 56 ciudades colombianas incluidas en este estudio; sin embargo, por razones relacionadas con el bajo número de pacientes en algunas de ellas, en los cuadros 4 y 5 solo se incluyeron las nueve mayores, donde residen $88,9 \%$ de las personas evaluadas, y se hizo el análisis para la sumatoria global. Se puede observar que las diferencias fueron significativas en la frecuencia de uso de monoterapia y en la comedicación entre las distintas ciudades. Los consumos de los grupos de antidepresivos se presentan, además, en forma de porcentaje de dosis diaria definida (DDD) (cuadro 5), para que puedan ser útiles en posteriores comparaciones. Se encontró que en pro-

CUADRO 2. Variables asociadas al tratamiento con monoterapia antidepresiva en modelos de regresión logística binaria, Colombia, 2010

\begin{tabular}{|c|c|c|c|c|c|c|c|}
\hline Variable & B & EE & Wald & $\mathrm{GL}$ & Sig & $\mathrm{RR}$ & IC 95\% \\
\hline \multicolumn{8}{|c|}{ Edad del paciente (ãnos) } \\
\hline 25 a 59 años & 0,035 & 0,060 & 0,343 & 1 & 0,558 & 1,036 & $0,921-1,164$ \\
\hline$\geq 60$ años & $-0,259$ & 0,062 & 17,441 & 1 & 0,000 & 0,772 & $0,684-0,872$ \\
\hline \multicolumn{8}{|c|}{ Ciudad del tratamiento } \\
\hline Bogotá & $-0,324$ & 0,054 & 35,560 & 1 & 0,000 & 0,723 & $0,650-0,804$ \\
\hline Medellín & 0,324 & 0,086 & 14,184 & 1 & 0,000 & 1,383 & $1,168-1,637$ \\
\hline Barranquilla & $-0,770$ & 0,156 & 24,379 & 1 & 0,000 & 0,463 & $0,341-0,629$ \\
\hline Bucaramanga & 0,664 & 0,119 & 31,028 & 1 & 0,000 & 1,942 & $1,538-2,454$ \\
\hline Cartagena & $-0,493$ & 0,162 & 9,229 & 1 & 0,002 & 0,611 & $0,444-0,839$ \\
\hline \multicolumn{8}{|l|}{ Sexo } \\
\hline Femenino & 0,107 & 0,056 & 3,676 & 1 & 0,055 & 1,113 & $0,998-1,242$ \\
\hline
\end{tabular}


CUADRO 3. Variables asociadas al tratamiento antidepresivo con comedicación en modelos de regresión logística binaria, Colombia, 2010

\begin{tabular}{lrrrrrrr}
\hline \multicolumn{1}{c}{ Variable } & B & EE & Wald & GL & Sig & RR & IC 95\% \\
\hline Edad del paciente (ãnos) & & & & & & & \\
$\quad$ < 10 & 2,137 & 0,542 & 15,526 & 1 & 0,000 & 8,470 & $2,926-24,516$ \\
11 a 25 & 1,295 & 0,174 & 55,587 & 1 & 0,000 & 3,652 & $2,598-5,133$ \\
25 a 59 & 0,295 & 0,064 & 21,148 & 1 & 0,000 & 1,344 & $1,185-1,524$ \\
$\quad$ 260 & $-0,784$ & 0,076 & 106,726 & 1 & 0,000 & 0,457 & $0,394-0,530$ \\
Ciudad del tratamiento & & & & & & & \\
$\quad$ Bogotá & $-0,131$ & 0,060 & 4,827 & 1 & 0,028 & 0,877 & $0,780-0,986$ \\
$\quad$ Cali & $-0,567$ & 0,125 & 20,640 & 1 & 0,000 & 0,567 & $0,444-0,724$ \\
Pereira & 0,074 & 0,106 & 0,485 & 1 & 0,486 & 1,077 & $0,875-1,325$ \\
$\quad$ Barranquilla & $-0,700$ & 0,173 & 16,390 & 1 & 0,000 & 0,497 & $0,354-0,697$ \\
Bucaramanga & 0,094 & 0,141 & 0,442 & 1 & 0,506 & 1,098 & $0,833-1,449$ \\
\hline
\end{tabular}

B: coeficiente de regresión; EE: error estándar; GL: grado de libertad; Sig: nivel de significación; RR: riesgo relativo; IC95\%: intervalo de confianza de $95 \%$

medio, se consumen 5,1 DDD de fluoxetina, 0,6 DDD de trazodona y 0,3 DDD de imipramina por 1000 habitantes día.

\section{DISCUSIÓN}

De los pacientes participantes en este estudio, alrededor de $50,0 \%$ son mayores de 60 años de edad (figura 1), cifra que no se corresponde con la bibliografía mundial, según la cual la mayor prevalencia de trastorno depresivo se observa en la población menor de 25 años $(2,6,10)$. Sin embargo, en algunos estudios se ha demostrado un aumento de la prevalencia en la población mayor de 60 años (5). En el presente estudio se encontraron algunos pacientes menores de 10 años tratados con terapia antidepresiva, como ha sido observado en otros países (32). Las mujeres reciben más comedicación que los varones, lo cual puede explicarse porque estos consultan menos y más tardíamente que las mujeres (30). Asimismo, la prevalencia de depresión en el sexo femenino es mayor, como lo demuestran algunos estudios $(3-5,7)$. Cabe destacar que la gran mayoría de los medicamentos analizados en este estudio están incluidos en el listado de medicamentos esenciales y que, como tales, poseen elevado valor intrínseco, de tal manera que son considerados como los grupos farmacológicos de elección en terapia antidepresiva y se estiman suficientes para el manejo de la depresión $(28,29)$. Al tratarse de un listado cerrado de medicamentos, se entiende por qué más de $91,8 \%$ de los pacientes son tratados con cuatro de los medicamentos incluidos en el mismo, aunque existe la opción de emplear medicamentos por fuera del listado, solicitados a través de la evaluación de un CTC. En lo correspondiente a los medicamentos disponibles para el tratamiento de los pacientes con depresión, la cifra de utilización de la fluoxetina, de $43,1 \%$, es inferior en comparación con los estudios que indican que los ISRS son los medicamentos más utilizados en la mayoría de los casos por sus beneficios en la reducción de la morbilidad por trastorno depresivo
(33). En segundo lugar, pero también empleada con frecuencia, está la trazodona, que ha demostrado una eficacia similar a la de otros antidepresivos (34). Es recomendable evaluar los posibles problemas asociados con la medicación de los pacientes, ya que se ha descrito con la utilización de estos dos antidepresivos un aumento del riesgo de suicidio, sobre todo en niños y adolescentes (35). En los Estados Unidos, para el año 2002 se estimaron 10,8 millones de prescripciones en jóvenes de entre $1 \mathrm{y}$ 17 años; los medicamentos más usados fueron los ISRS, especialmente en trastornos de ansiedad y del ánimo, donde más del $60,0 \%$ de dichas prescripciones fueron realizadas por psiquiatras (32). Sin embargo, nuestro estudio encontró una baja proporción de prescriptores psiquiatras en todos los grupos de edad; la causa de este hecho se desconoce, pero puede estar asociada a dificultades de acceso a tal servicio en el SGSSS o a subregistro.

Las dosis prescritas de los antidepresivos analizados (cuadro 1) se encuentran por debajo del rango de la dosificación recomendada como efectiva para la mayoría de estos, con excepción de la fluoxetina (10). Las diferencias halladas entre los pacientes tratados con monoterapia y aquellos que reciben politerapia se pueden explicar porque se dispone de pocas opciones de medicamentos y algunas asociaciones revisten riesgo, porque se trata de pacientes bien controlados con los antidepresivos usuales, porque la psicoterapia de apoyo coadyuvante es efectiva $\mathrm{o}$, incluso, porque se están utilizando otros medicamentos con indicaciones no aprobadas (off label) para lograr el control de la enfermedad.

El empleo de dosis menores que las recomendadas en los casos de la tra-

CUADRO 4. Comparación de algunas variables demográficas e indicadores de prescripción de antidepresivos entre nueve ciudades colombianas, 2010

\begin{tabular}{|c|c|c|c|c|c|c|c|c|c|c|}
\hline Variable/indicador & $\begin{array}{c}\text { Bogotá } \\
n=4407\end{array}$ & $\begin{array}{c}\text { Manizales } \\
n=1053\end{array}$ & $\begin{array}{c}\text { Medellín } \\
n=792\end{array}$ & $\begin{array}{c}\text { Cali } \\
n=700\end{array}$ & $\begin{array}{l}\text { Pereira } \\
n=642\end{array}$ & $\begin{array}{c}\text { Barranquilla } \\
\quad n=371\end{array}$ & $\begin{array}{c}\text { Bucaramanga } \\
\quad n=328\end{array}$ & $\begin{array}{c}\text { Cartagena } \\
n=276\end{array}$ & $\begin{array}{l}\text { Ibagué } \\
n=220\end{array}$ & $\begin{array}{c}\text { Global } \\
n=9881\end{array}$ \\
\hline Edad promedio & 61,1 & 57,9 & 57,5 & 60,5 & 55,0 & 59,6 & 56,1 & 52,1 & 59,1 & 59,2 \\
\hline Mujeres (\%) & 73,1 & 78,1 & 78,3 & 69,9 & 74,8 & 70,1 & 67,1 & 76,1 & 75,5 & 73,8 \\
\hline Monoterapia (\%) & 80,8 & 76,3 & 68,2 & 76,4 & 75,4 & 86,3 & 60,1 & 82,2 & 73,2 & 76,9 \\
\hline Comedicación (\%) & 83,1 & 82,3 & 80,8 & 87,7 & 77,2 & 88,9 & 77,4 & 80,8 & 78,6 & 81,9 \\
\hline \multicolumn{11}{|c|}{ Tasas de prescripción (\%) } \\
\hline Fluoxetina & 29,4 & 51,2 & 56,4 & 51,7 & 50,6 & 35,0 & 47,9 & 55,1 & 44,1 & 40,4 \\
\hline Trazodona & 42,5 & 43,5 & 24,5 & 32,9 & 31,0 & 23,7 & 33,8 & 9,4 & 39,5 & 36,7 \\
\hline Amitriptilina & 29,2 & 13,9 & 21,3 & 22,6 & 16,8 & 34,8 & 16,8 & 23,6 & 24,1 & 24,4 \\
\hline Imipramina & 4,6 & 3,1 & 10,6 & 6,7 & 10,6 & 9,7 & 8,8 & 9,4 & 4,5 & 6,1 \\
\hline Fluoxetina (jarabe) & 4,3 & 0,9 & 2,2 & 2,0 & 2,6 & 0,8 & 0,3 & 0,0 & 2,7 & 2,7 \\
\hline
\end{tabular}


CUADRO 5. Relación entre la dosis media administrada y la dosis diaria definida (DDD) de antidepresivos en nueve ciudades colombianas, 2010

\begin{tabular}{|c|c|c|c|c|c|c|c|c|c|c|}
\hline Medicamento & $\begin{array}{c}\text { Bogotá } \\
n=4407\end{array}$ & $\begin{array}{c}\text { Manizales } \\
n=1053\end{array}$ & $\begin{array}{c}\text { Medellín } \\
n=792\end{array}$ & $\begin{array}{c}\text { Cali } \\
n=700\end{array}$ & $\begin{array}{l}\text { Pereira } \\
n=642\end{array}$ & $\begin{array}{c}\text { Barranquilla } \\
\quad n=371\end{array}$ & $\begin{array}{c}\text { Bucaramanga } \\
\quad n=328\end{array}$ & $\begin{array}{c}\text { Cartagena } \\
n=276\end{array}$ & $\begin{array}{l}\text { lbagué } \\
n=220\end{array}$ & $\begin{array}{c}\text { Global } \\
n=9881\end{array}$ \\
\hline Fluoxetina & 1,3 & 1,3 & 1,5 & 1,2 & 1,3 & 1,4 & 1,5 & 1,5 & 1,6 & 1,3 \\
\hline Trazodona & 0,2 & 0,2 & 0,2 & 0,2 & 0,2 & 0,2 & 0,2 & 0,3 & 0,2 & 0,2 \\
\hline Amitriptilina & 0,4 & 0,4 & 0,4 & 0,4 & 0,4 & 0,3 & 0,4 & 0,4 & 0,5 & 0,4 \\
\hline Imipramina & 0,7 & 0,3 & 0,5 & 0,6 & 0,5 & 0,3 & 0,5 & 0,4 & 0,5 & 0,5 \\
\hline
\end{tabular}

zodona, la amitriptilina y la imipramina puede interpretarse de tres maneras: los pacientes están controlados y requieren dosis menores; los pacientes están subdosificados, con riesgo de no conseguir un adecuado control de su trastorno; o los medicamentos pueden estar siendo utilizados en alguna indicación diferente a la aprobada (12). Se recomienda hacer estudios que indaguen sobre las posibles explicaciones a este fenómeno. Además se encontró, aunque en bajo porcentaje, algunos pacientes que recibían concomitantemente cuatro e, incluso, cinco psicofármacos, una conducta que puede considerarse poco racional y que reviste un riesgo adicional de interacciones que modifiquen la respuesta farmacológica.

$\mathrm{Al}$ inferir la comorbilidad a partir de la comedicación prescrita, se encontró que la hipertensión y el hipotiroidismo son las enfermedades más prevalentes, con $52,3 \%$ y $23,3 \%$ de los individuos, respectivamente; estas cifras son similares a las encontradas en otros estudios (16, 20). Por otra parte, en estos pacientes se debe tener precaución con las posibles interacciones farmacológicas, que pueden alterar el control de alguna de las comorbilidades. Del total de participantes, $15,4 \%$ son tratados simultáneamente con anticonvulsivos, lo cual indica una alta prevalencia de enfermedad epiléptica o un uso posiblemente inadecuado de estos medicamentos, ya que los antidepresivos tricíclicos pueden reducir el umbral convulsivo (12). Las otras comorbilidades se presentaron en menor proporción, pero son evidencia de que el trastorno depresivo se puede asociar a otras enfermedades que afectan la calidad de vida de los pacientes. Como se desconocen las razones que expliquen las diferencias halladas entre ciudades en cuanto a la frecuencia de empleo de comedicación, se recomienda hacer estudios ulteriores que proporcionen herramientas útiles para reducir el riesgo de interacciones.

El grupo de pacientes con terapia combinada de fluoxetina más trazodona, y en especial aquellos que reciben más de dos antidepresivos, debe ser objeto de un seguimiento especial para detectar y reducir de manera oportuna el riesgo de síndrome serotoninérgico, una complicación ampliamente descrita que puede causar grave morbilidad (10). La presencia de estos casos debe obligar a la evaluación individual de los pacientes para determinar si tal asociación está justificada o no, de acuerdo con la evolución y severidad del cuadro clínico, a fin de minimizar los posibles daños.

Las diferencias encontradas en los patrones de prescripción entre las distintas ciudades colombianas del estudio, tales como las frecuencias de utilización de algunos antidepresivos (cuadros 4 y 5) no son sorprendentes ya que la variabilidad en la atención médica, en particular en los hábitos de prescripción, es un hallazgo constante en los estudios farmacoepidemiológicos (30). No obstante, fue común que en todas las ciudades se emplearan DDD inferiores a las recomendadas para algunos medicamentos. Estas variaciones, de importancia por sus implicaciones médicas, sociales y económicas, están relacionadas con estilos de práctica profesional que también resultan de factores personales, de formación y de capacitación de los médicos (36).

Esta investigación presenta ciertas limitaciones para la interpretación de algunos resultados debido a que la información fue obtenida de bases de datos y no directamente del paciente o el prescriptor, y a que no se consultó la historia clínica. Estas limitaciones pueden superarse con otros estudios de utilización de medicamentos correspondientes a la segunda fase de esta línea de investigación, que permitan caracterizar la prescripción de antidepresivos. Como el diseño solo permite recoger los datos de la dispensación, se hace necesario en estudios ulteriores recopilar la información acerca de la clasificación de la depresión en estos pacientes, los intervalos de dosis empleados, la incidencia de reacciones adversas atribuibles a la medicación, el cumplimiento terapéutico, el grado de control de la depresión y la morbilidad asociada. Por otra parte, dado que no se evaluaron los valores iniciales ni los de control de este trastorno, se desconoce incluso si la prescripción era necesaria o adecuada. Cabe tomar en cuenta que al tratarse de una población cautiva y que recibe medicamentos incluidos en un listado específico, las conclusiones son aplicables exclusivamente a poblaciones que presenten características similares a ésta.

A partir de los patrones de prescripción encontrados en este estudio puede afirmarse que, en general, predominan los hábitos de prescripción de medicamentos incluidos en el listado de medicamentos esenciales del país, principalmente en monoterapia antidepresiva -en especial, con ISRS-, y con el respaldo científico de las guías de tratamiento de la American Psychiatric Association. La fluoxetina se prescribe en dosis adecuadas, y los otros medicamentos en dosis bajas. Pero se encontró un número importante de pacientes que reciben dos y hasta tres y cuatro antidepresivos asociados; la causa de este tipo de prescripción se desconoce, pero pone a los pacientes en riesgo de síndrome serotoninérgico. La hipertensión, el hipotiroidismo, el dolor y la epilepsia fueron las comorbilidades más comunes y la presencia de estas enfermedades debe hacer pensar al médico en detectar una posible depresión simultánea. El empleo de dosis inferiores a las recomendadas muestra la necesidad de indagar en todas las posibles indicaciones y usos de estos medicamentos $\mathrm{y}$, cuando se emplean en el trastorno depresivo, requieren de intervenciones destinadas a mejorar los criterios de prescripción en los pacientes afiliados al SGSSS de Colombia.

Es necesario brindar educación permanente para garantizar que los médicos estén actualizados en el manejo de la depresión, utilicen los medicamentos indicados, empleen las asociaciones cuando 
sea necesario, y siempre a las dosis recomendadas para alcanzar el control de la enfermedad. Se debe hacer un estudio de efectividad del tratamiento antidepresivo en este mismo grupo de pacientes para evaluar los resultados que se obtienen a las dosis usadas actualmente en la depresión, valorando, al mismo tiempo, la pertinencia de la comedicación que reciben los pacientes.

\section{REFERENCIAS}

1. Organización Mundial de Salud. Estadísticas sanitarias mundiales 2007. Parte 1. Diez cuestiones estadísticas de especial interés en la salud pública mundial. Disponible en: http:/ / www.who.int/whosis/whostat/ WHS2007Sp_Parte1.pdf Acceso 28 de septiembre de 2009.

2. Torres Y, Montoya D. II estudio nacional de salud mental y consumo de sustancias psicoactivas, Colombia 1997. República de Colombia, Ministerio de salud. Santafé de Bogotá. 1998;175-96.

3. Saraceno B, Shekhar S, Caraveo-Adnuaga JJ, Kohn R, Levav I, Caldas de Almeida JM, et al. Los trastornos mentales en América Latina y el Caribe: Asunto prioritario para la salud pública. Rev Panam Salud Publica. 2005;18:229-40.

4. Hasin DS, Goodwin RD, Stinson FS, Grant BF. Epidemiology of major depressive disorder: results from the National Epidemiologic Survey on Alcoholism and Related Conditions. Arch Gen Psychiatry. 2005;62:1097-106.

5. Belló M, Puentes-Rosas E, Medina-Mora ME, Lozano R. Prevalencia y diagnóstico de depresión en población adulta en México. Salud Publica Mex. 2005; 47 supl 1:S4-S11.

6. Kaplan H, Sadock B. Tratado de psiquiatría, $6^{a}$ ed. Buenos Aires: Intermédica; 1997. v.2.

7. Posada Villa J, Gómez Gutiérrez LF, Gómez Serrano LC. Estudio nacional de salud mental, Colombia 2003. República de Colombia, Ministerio de protección social. Cali. 2005; 24-6.

8. Saiz González D, Rodríguez M, García C, Prieto R, Saiz-Ruiz J. Diferencias de sexo en el trastorno depresivo mayor: síntomas somáticos y calidad de vida. Rev Psiquiatr Salud Ment (Barc.). 2009;2:119-27.

9. Kendler K, Karkowski L, Prescott C. Causal relationship between stressful life events and the onset of major depression. Am J Psychiatry. 1999;1:837-41

10. Sadock B, Sadock V. Sinopsis de Psiquiatría, $10^{a}$ ed., Baltimore: Williams and Wilkins; 2009. Pp. 534-78.

11. Baldessarini R. Drug therapy of depression and anxiety disorders. En: Brunton LL, Lazo JS, Parker KL, eds. Goodman and Gilman's. The Pharmacological Basis of Therapeutics, $11^{\text {a }}$ ed. Madrid:McGraw-Hill; 2006. Pp. 429-59.

12. American Psychiatric Association. Practice Guideline for the Treatment of Patients With Major Depressive Disorder, 3rd Ed. Disponible en http://www.psychiatryonline. com/pracGuide/pracGuideTopic_7.aspx Acceso 2 de diciembre de 2010.

13. Nicholson A, Kuper H, Hemingway H. Depression as an aetiologic and prognostic factor in coronary heart disease: a meta-analysis of 6,362 events among 146,538 participants in 54 observational studies. Eur Heart J. 2006;27:2763.

14. Pihl E, Jacobsson A, Fridlund B, Stromberg A, Martensson J. Depression and health-related quality of life in elderly patients suffering from heart failure and their spouses: a comparative study. Eur J Heart Fail. 2005;7:583-7.

15. Carod-Artal FJ, Gonzalez-Gutierrez JL, EgidoHerrero JA, Varela-de Seijas E. Depresión post-ictus: factores predictivos al año de seguimiento. Rev Neurol. 2002;35:101-6.

16. Dois CA, Cazenave. Pesquisa de depresión mayor en hipertensos consultantes de la atención primaria. Rev Med Chile. 2009;137: $475-80$.

17. Massie MJ. Prevalence of Depression in Patients With Cancer. J Natl Cancer Inst Monogr. 2004;32:57-71.

18. Farabaugh AH, Locascio JJ, Yap L, Weintraub D, McDonald WM, Agoston M, et al. Pattern of depressive symptoms in Parkinson's disease. Psychosomatics. 2009;50:448-54

19. Wolff LC, Alvarado MR, Wolff RM. Prevalencia, factores de riesgo y manejo de la depresión en pacientes con infección por VIH: Revisión de la literatura. Rev Chilena Infectol. 2010;27:65-74.

20. Valdivieso FS, Kripper C, Ivelic JA, Fardella C, Gloger S, Quiroz D. Alta prevalencia de disfunción tiroidea en pacientes psiquiátricos hospitalizados. Rev Med Chile. 2006;134:623-8

21. Anderson RJ, Freedland KE, Clouse RE Lustman PJ. The prevalence of comorbid depression in adults with diabetes: A metaanalysis. Diabetes Care. 2001;24:1069:78.

22. Ismail $\mathrm{K}$, Winkley $\mathrm{K}$, Stahl $\mathrm{D}$, Chalder $\mathrm{T}$, Edmonds M. A cohort study of people with diabetes and their first foot ulcer: the role of depression on mortality. Diabetes Care. 2007;30:1473-9.

23. Caballero-Uribe CV, Venegas C, Padilla A, Paternina P, Peña R, Peñuela M. Depresión, estrés y desesperanza en pacientes con artritis reumatoide del Caribe colombiano. Rev Colomb Reumatol. 2004;11:300-5.

24. Chacón Narvaez V, Muñoz Anduquia CX, Rivas Nieto JC, Miranda Bastidas CA. Estudio descriptivo de la prevalencia de depresión en pacientes con epilepsia que consultan el Hospital Psiquiátrico Universitario del Valle (HPUV) y la Liga contra la Epilepsia (LCE) en Cali, Colombia. Rev Col Psiquiatria. 2007;36:31-40.

25. Baynes D, Mulholland C, Cooper SJ Montgomery RC, MacFlynn G, Lynch G, et al. Depressive symptoms in stable chronic schizophrenia: prevalence and relationship to psychopathology and treatment. Schizophr Res. 2000;45:47-56.

26. Pini $S$, Cassano GB, Simonini E, Savino M, Russo A, Montgomery SA. Prevalence of anxiety disorders comorbidity in bipolar de-
Financiación. El trabajo de investigación recibió financiación por parte de la Universidad Tecnológica de Pereira y de Audifarma S.A. pression, unipolar depression and dysthymia. Journal of Affective Disorders. 1997;42:14553.

27. Buysse DJ, Angst J, Gamma A, Ajdacic V, Eich D, Rössler W. Prevalence, course, and comorbidity of Insomnia and depression in young adults. SLEEP. 2008;31:473-80

28. Ministerio de Salud de Colombia. Consejo Nacional de Seguridad Social en Salud. Acuerdo 228 de 2002. Disponible en http:// www.col.ops-oms.org/medicamentos/ Documentos/ac228_02.htm Acceso 1 de noviembre de 2009.

29. Ministerio de Salud de Colombia. Resolución 5061 de 1997. Disponible en http:// www.medicolegal.com.co/ $/ \mathrm{rml} /$ files $\% 5$ Cnoticias\%5Ccomit_medicamen_resolu 5061.pdf Acceso 1 de noviembre de 2009.

30. Machado JE, Moncada JC, Mesa G. Prescription patterns for antilipidemic drugs in a group of Colombian patients. Rev Panam Salud Publica. 2008;23:179-87.

31. Ministerio de Salud de Colombia. Dirección de Desarrollo Científico y Tecnológico. Normas Científicas, Técnicas y Administrativas para la Investigación en Salud. Resolución No. 8430 de 1993. Disponible en http:/ / www. minproteccionsocial.gov.co/vbecontent/ library/documents/DocNewsNo267711.pdf Acceso 1 de noviembre de 2009.

32. Leslie LK, Newman TB, Chesney PJ, Perrin JM. The Food and Drug Administration's deliberations on antidepressant use in pediatric patients. Pediatrics. 2005;116:195-204.

33. Cruz I, Serna C, Real J, Rué M, Soler J, Galván L. Comparison of the consumption of antidepressants in the immigrant and native populations in a Spanish health region: an observational study. BMC Public Health. 2010;10:255.

34. Gartlehner G, Hansen RA, Thieda P, DeVeaugh-Geiss AM, Gaynes BN, Krebs EE, et al. Comparative Effectiveness of SecondGeneration Antidepressants in the Pharmacologic Treatment of Adult Depression [Internet]. Rockville (MD): Agency for Healthcare Research and Quality (US); 2007 Jan. Report No: 07-EHC007-EF.AHRQ Comparative Effectiveness Reviews.

35. Jick SS, Dean AD, Jick H. Antidepressants and suicide. BMJ. 1995;310:215-8.

36. Gómez J, Candás M, Fidalgo G, Armesto J, Calvo M, de Marino M, et al. Análisis del consumo de medicamentos utilizando indicadores de calidad en la prescripción. Aten Primaria. 2000;25:618-24.

Manuscrito recibido el 6 de enero de 2011. Aceptado para publicación, tras revisión, el 2 de junio de 2011. 
ABSTRACT Objective. Determine patterns of antidepressive drug prescription in a group of patients affiliated with the General Social Security Health System in Colombia.

Methods. Observational descriptive study of 9881 patients, of both sexes and older

Antidepressant prescription patterns in patients affiliated with the General Social Security Health System of Colombia than 5 years of age, medicated with antidepressants and continuously treated from August to October 2009. The patients include residents from 56 Colombian cities. A database was designed based on the consumption of medicines obtained from the company that distributes them to the patients.

Results. The average age was $59.1 \pm 16.1$ years; $73.7 \%$ of the participants were women. Of the total number of patients, $83.3 \%$ were treated with monotherapy and $16.7 \%$ with two or more antidepressants. The order of the prescription of the medicines was: selective serotonin reuptake inhibitors, $47.0 \%$; atypical, 37.8\%; tricyclical, $31.8 \%$; selective serotonin reuptake inhibitors and norepinephrine, $1.8 \%$; and selective norepinephrine reuptake inhibitors, $0.03 \%$. The combinations most used were fluoxetine + trazodone $(n=1029)$; amitriptyline + fluoxetine $(n=265)$; amitriptyline + trazodone $(n=122)$; fluoxetine + imipramine $(n=106)$; and imipramine + trazodone $(n=71)$. The most prescribed co-medications were anti-hypertensives $(52.3 \%)$; thyroid hormones (23.3\%); anti-inflammatories (19.6\%); anti-epileptics (15.4\%); anti-diabetics $(13.8 \%)$; anti-anxiety and hypnotics $(12.4 \%)$; antipsychotics $(7.4 \%)$; anti-Parkinsons $(4.3 \%)$; and anti-neoplastics (2.2\%).

Conclusions. The practice of prescribing medicines with a high therapeutic value predominates, mainly for antidepressive monotherapy. Most of the antidepressants are prescribed at dosages lower than those recommended. There is a need to design educational strategies to correct some prescription practices and to conduct research

Key words Depressive disorder; antidepressive agents; drug prescriptions; amitriptyline; fluoxetine; imipramine; trazodone; Colombia. 